\title{
¿Qué explica la Física sobre la influencia de la Luna en la Tierra? Una propuesta de enseñanza
}

\author{
Jordi Solbes (D), José Cantó (D) , Olga Mayoral (D) , Tatiana Pina (D) \\ Departamento de Didáctica de las Ciencias Experimentales y Sociales. Universitat de València. España. \\ jordi.solbes@uv.es,jose.canto@uv.es,olga.mayoral@uv.es,tatiana.pina@uv.es
}

[Recibido: 9 abril 2021. Revisado: 4 junio 2021. Aceptado: 7 julio 2021]

\begin{abstract}
Resumen:. En este trabajo se abordan ideas pseudocientíficas en torno a la influencia de la Luna en nuestro planeta. Para ello se analiza si los libros de Física de Bachillerato y de Educación Superior tratan la influencia de la gravedad de la Luna en la Tierra y cómo lo hacen. Para el análisis se utiliza una muestra de 17 libros de textos de Física de Bachillerato y 17 utilizados en cursos de ciencia e ingeniería (13 de Física, 2 de Óptica y 2 de Astronomía) de distintos años de publicación. Los resultados revelan la escasa atención que prestan estos libros a los temas relacionados con la física de la Luna y su influencia sobre nuestro planeta, así como un enfoque descontextualizado de la enseñanza de la Física. A continuación, se presenta una propuesta para introducir correctamente los aspectos básicos sobre la física de la Luna (gravitación, mareas, luminosidad) que refuta las supuestas influencias de la Luna en los seres vivos.
\end{abstract}

Palabras clave: Enseñanza de la Física; Física de las mareas; concepciones pseudocientíficas sobre la influencia de la Luna.

Does How Physic explain Moon's influence on Earth? A teaching proposal

Abstract: This paper addresses pseudoscientific ideas regarding the influence of the Moon on our planet. To do so, a study is made of whether and how high school and higher education physics textbooks deal with the influence of the Moon's gravity on Earth. A sample of 17 High School physics textbooks and 17 textbooks used in science and engineering courses (13 in Physics, 2 in Optics and 2 in Astronomy) from different years of publication are used for the analysis. The results reveal that these books pay little attention to topics related to the physics of the Moon and its influence on our planet, as well as a decontextualized approach to the teaching of Physics. This is followed by a proposal for a correct introduction to basic aspects of the Moon's physics (gravitation, tides, luminosity) that refutes the Moon's supposed influences on living things.

Keywords: Physics teaching; tidal physics; pseudo-scientific conceptions of the influence of the Moon.

Para citar este artículo: Solbes, J., Cantó, J., Mayoral, O. y Pina, T. (2021) ¿Qué explica la Física sobre la influencia de la Luna en la Tierra? Una propuesta de enseñanza. Revista Eureka sobre Enseñanza y Divulgación de las Ciencias 18(3), 3701. doi: 10.25267/Rev_Eureka_ensen_divulg_cienc.2021.v18.i3.3701

\section{Introducción}

La gravedad de la Luna y, en concreto, su evidente influencia en las mareas ha sido una de las razones por las que la cultura popular ha atribuido a nuestro satélite (en particular, a alguna de sus fases) influencia sobre una serie de fenómenos que nada tienen que ver con ella. La posible influencia de la Luna en las personas ha sido una de las cuestiones más estudiadas debido al interés que suscita. Destacan estudios de ámbito científico aplicados a la medicina y psiquiatría clínica, como por ejemplo el realizado por Arliss, Kaplan y Galvin (2005), quienes analizaron 564.039 partos entre 1997 y 2001, a lo largo de 62 ciclos lunares, y no encontraron ninguna relación con el ciclo lunar. O el de Marco-Gracia (2019), quien estudió 23.689 nacimientos repartidos en 1484 ciclos lunares entre 1810 y 1929 en la España rural, y no encontró evidencia científica de que las fases lunares influyesen. Sin embargo, hay acontecimientos singulares que sí que influyen en el número de partos, como son las victorias en eventos deportivos, tras las que se observa un repunte de partos (Montesinos et al. 2013). 
Con respecto a la salud mental, destaca el metaanálisis de Rotton y Kelly (1985), realizado a partir de 37 estudios, que examinó las relaciones entre las fases de la Luna y varios tipos de problemas mentales, incluidos ingresos hospitalarios, trastornos psiquiátricos, llamadas de crisis, homicidios y otros delitos penales. Aunque surgieron algunas relaciones estadísticamente significativas, las estimaciones del tamaño del efecto indicaron que las fases de la Luna representaban no más del 1\% de la varianza de las actividades que se suelen denominar lunáticas. Un estudio más reciente (Gupta, Nolan, Bux y Schneeberger 2019) evaluó las tasas de admisión y alta del tratamiento psiquiátrico hospitalario, así como la duración de la estancia, en relación con el ciclo lunar en un total de 17.966 casos concluyendo que no se aportaban pruebas de que la Luna influya en nuestro estado mental.

También se ha promovido la idea de que las fases lunares influyen en el crecimiento y fortaleza del cabello humano y de las uñas. No obstante, diversos estudios demuestran que el crecimiento del cabello $(1,5 \mathrm{~cm}$ al mes) sólo está provocado por la actividad del folículo piloso (Messenger 1983) y el de las uñas principalmente por las capas germinativas de su matriz (Lipner y Scher 2017), no existiendo ninguna relación entre el crecimiento y las fases lunares.

En cuanto a la influencia de la Luna en la agricultura, el arraigo de las creencias populares es mucho mayor y persistente dada la enorme cantidad de variables que intervienen y que dificultan un abordaje sencillo. Tradicionalmente se cree que la Luna influye en el crecimiento de las plantas (en el transporte de la savia, en la fotosíntesis, ...) y que, por ello, las fases lunares deben ser tenidas en cuenta para organizar las diferentes labores agrícolas. La idea subyacente es que si la Luna es capaz de producir mareas, es decir, de mover el agua de los mares abiertos y grandes océanos, también puede mover la savia de las plantas. Y en este sentido, Restrepo (2004) llega a afirmar que la savia asciende por los vasos conductores hacia las hojas durante la Luna llena (marea viva) y que desciende hacia las raíces en Luna nueva (marea muerta), por lo que se recomiendan unas tareas agrícolas determinadas en cada una de las fases (Anglés 1984). Sin embargo, si eso fuera así, la savia también debería subir y bajar dos veces al día siguiendo el mismo patrón diario que las mareas.

La creencia popular también considera relevante la luminosidad de la Luna en la fotosíntesis de las plantas, si bien el rango de radiación fotosintéticamente activa (RFA) que emite la Luna llena es inferior al que requieren las plantas para activar sus fotosistemas (Raven y Cockell 2006). En ese sentido, Mayoral, Solbes, Cantó y Pina (2020) realizaron una revisión de casi un centenar de artículos científicos y seis manuales universitarios de Física y seis de Botánica con la finalidad de analizar qué decían la ciencia consolidada y las investigaciones científicas con relación a la posible influencia de la Luna en el crecimiento de las plantas. Los resultados de este trabajo muestran que en las monografías no se establece ninguna relación entre la Luna y el crecimiento de las plantas y que en los artículos científicos no se aporta ninguna evidencia científica clara que permita corroborar la influencia del satélite de la Tierra en los cultivos.

El presente artículo pretende abordar las ideas pseudocientíficas en torno a la influencia de la Luna en nuestro planeta a través de un doble enfoque. Por un lado, analizando manuales de Física, tanto de Bachillerato como universitarios, para valorar si en ellos se aborda la influencia de la gravedad de la Luna en la Tierra y cómo se hace, prestando especial atención a la detección de posibles enfoques incorrectos en la enseñanza de la Física. Y, por otro lado, desarrollando una propuesta didáctica que aborde correctamente los aspectos básicos sobre la física de la Luna (gravitación, mareas, luminosidad) y que permita refutar las supuestas influencias de la Luna en los seres vivos. 


\section{Análisis de manuales de Física}

Partiendo de la base de que una de las fuentes del conocimiento científico radica en la formación escolar, cabe preguntarse qué información ofrecen los manuales empleados en la educación científica con relación a los efectos de la Luna en nuestro planeta. Un trabajo reciente (Armario, Jiménez-Tenorio y Oliva 2021) analiza 18 libros de texto de Educación Secundaria Obligatoria de Física y Química, y Biología y Geología, encontrando que el tema de las mareas solo es tratado en libros de Biología y Geología. En este trabajo nos centramos exclusivamente en los libros de Física, ya que se trata del único campo de conocimiento que permite efectuar los cálculos que aporten pruebas mesurables de la magnitud de la influencia o no de la Luna en nuestro planeta. En este apartado mostramos los resultados del análisis de 17 libros de texto de Física de Bachillerato (publicados entre 1961 y 2016) y 13 manuales de Física, 2 de Astronomía y 2 de Óptica universitarios (publicados entre 1971 y 2009) en relación con la fuerza gravitacional de la Luna sobre la Tierra, las mareas y la magnitud de la iluminación de la Luna en la Tierra.

El estudio se ha realizado sobre libros editados en diferentes años y editoriales para determinar en qué medida el paso del tiempo ha podido influir en su inclusión o no, así como los posibles tratamientos o enfoques. Para la selección se tuvo en cuenta la frecuencia de uso en los centros educativos o universitarios, así como su disponibilidad. Para la recogida de datos se establecieron una serie de palabras clave (p. ej. gravedad, mareas, Luna, iluminancia, plantas) que debían aparecer en el índice temático o en el glosario. Posteriormente se revisó el contenido de las páginas del tema o capítulo en el que se incluían estas palabras. Esta información se recogió en una tabla junto con los datos identificativos del manual y las páginas de referencia.

El primer resultado que llama la atención de los 17 libros analizados de Física es que, si bien no existe ninguna mención a la luminosidad de la Luna, sí que han sido abordadas las cuestiones de gravitación, tal como se muestra en la tabla 1.

Tabla 1. Manuales de Física analizados utilizados en Bachillerato. Se indican los contenidos que abordan cuestiones relativas a la Luna y la gravitación.

\begin{tabular}{|l|l|}
\hline \multicolumn{1}{|c|}{ Libros de Bachillerato o equivalentes } & \multicolumn{1}{|c|}{ Contenidos relacionados con la Luna } \\
\hline $\begin{array}{l}\text { Marcos C., Martínez J. (1961) Física } 6^{\circ} \text { curso. Madrid: } \\
\text { SM }\end{array}$ & Ley de gravitación universal \\
\hline Marcos C., Martínez J. (1961) Física. Preu. Madrid: SM & Energía potencial gravitatoria \\
\hline Pujal M. (1961) Física preuniversitaria. Barcelona: Teide & Energía potencial gravitatoria \\
\hline Pérez A. (1972) Física 6 ${ }^{\circ}$ curso. Alcoy: Marfil & Ley de gravitación universal \\
\hline $\begin{array}{l}\text { Pérez A., Miralles L. (1965) Física y su metodología. } \\
\text { Magisterio. Alcoy: Marfil }\end{array}$ & Ley de gravitación universal \\
\hline $\begin{array}{l}\text { Caturla E., Vidal F. (1989) Quark. Física COU. } \\
\text { Barcelona: Vicens Vives }\end{array}$ & $\begin{array}{l}\text { Ley de gravitación universal, deducción a partir de la } \\
\text { misma de las leyes de Kepler }\end{array}$ \\
\hline $\begin{array}{l}\text { Tejerina F. (coord.); Candel A., Satoca J., Soler J.B., } \\
\text { Tent J.J. (1988) Física COU. Madrid: Anaya }\end{array}$ & $\begin{array}{l}\text { Ley de gravitación universal, campo gravitatorio y } \\
\text { eléctrico }\end{array}$ \\
\hline $\begin{array}{l}\text { Solbes J., Tarín F. (1996) Física } 2^{\circ} \text { Bachillerato. } \\
\text { Barcelona: Octaedro }\end{array}$ & Ley de gravitación universal, campo gravitatorio \\
\hline $\begin{array}{l}\text { Martín J., Fraile J.M., Alonso A. (1998) Física I. } \\
\text { Barcelona: Santillana }\end{array}$ & Ley de gravitación universal \\
\hline
\end{tabular}


Tabla 1. Continuación

\begin{tabular}{|c|c|}
\hline Libros de Bachillerato o equivalentes & Contenidos relacionados con la Luna \\
\hline $\begin{array}{l}\text { Martín J., Ruiz E., Fraile J.M. (1999) Física II. } \\
\text { Barcelona: Santillana }\end{array}$ & $\begin{array}{l}\text { Campo gravitatorio, energía total incluyendo Luna, } \\
\text { nave espacial alrededor de Luna }\end{array}$ \\
\hline $\begin{array}{l}\text { Dalmau J.F., Pérez M., Satoca J., Tejerina F. (2007) } \\
\text { Física } 2^{\circ} \text { Bachillerato. Madrid: Anaya }\end{array}$ & $\begin{array}{l}\text { Ley de gravitación universal, campo gravitatorio, } \\
\text { mareas }\end{array}$ \\
\hline $\begin{array}{l}\text { Lorente S., Sendra F., Enciso E., Quílez J., Romero } \\
\text { J. (2009) Éter. Física. Valencia: ECIR }\end{array}$ & $\begin{array}{l}\text { Ley de gravitación universal, campo gravitatorio, } \\
\text { mareas (actividad complementaria) }\end{array}$ \\
\hline $\begin{array}{l}\text { Andrés D.C., Antón J.L., Barrio J. (2009) Física } 2^{\circ} \\
\text { Bachillerato. Madrid: Editext }\end{array}$ & $\begin{array}{l}\text { Ley de gravitación universal, campo gravitatorio, } \\
\text { mareas con fuerza centrífuga }(\mathrm{Fc})\end{array}$ \\
\hline $\begin{array}{l}\text { Ruiz F., Tarín F. (2009) Física } 2^{\circ} \text { Bachillerato. Madrid: } \\
\text { McGraw-Hill }\end{array}$ & Ley de gravitación universal, campo gravitatorio \\
\hline $\begin{array}{l}\text { Martínez E., Martínez, J. (2016) Física } 2^{\circ} \text { Bachillerato. } \\
\text { Madrid: Paraninfo }\end{array}$ & $\begin{array}{l}\text { Campo gravitatorio a } 100 \mathrm{~km} \text { de la superficie de la } \\
\text { Luna }\end{array}$ \\
\hline $\begin{array}{l}\text { Nacenta P., Romo N., Trueba J.L., Puente J. (2016) } \\
\text { Física } 2^{\circ} \text { Bachillerato. Madrid: SM }\end{array}$ & $\begin{array}{l}\text { Ley de gravitación universal, aceleración de la } \\
\text { gravedad en superficie }\end{array}$ \\
\hline $\begin{array}{l}\text { Vidal M.C., Sánchez, D. (2016) Física } 2^{\circ} \text { Bachillerato. } \\
\text { Madrid: Santillana Educación }\end{array}$ & $\begin{array}{l}\text { Ley de gravitación universal, campo gravitatorio, } \\
\text { mareas con } \mathrm{Fc}\end{array}$ \\
\hline
\end{tabular}

También se observa que en los libros más antiguos sólo se introduce la Ley de gravitación universal o la energía potencial gravitatoria mientras que, en los más recientes, se incluyen ambos conceptos además del de campo gravitatorio (en general, el terrestre y el de la Luna en su superficie). Y, sin embargo, tan solo cuatro manuales abordan la gravedad de la Luna sobre la Tierra y las mareas, tal y como se comenta a continuación. De estos manuales, tres se corresponden con la Ley Orgánica de Educación (LOE), porque en el currículo de la Comunidad Valenciana (y no en el estatal), aparecía el contenido "Explicación de las mareas", y el último manual, con la Ley Orgánica para la Mejora de la Calidad Educativa (LOMCE) (Tabla 1).

El manual de Lorente, Sendra, Enciso, Quílez y Romero (2009), en una actividad complementaria, ofrece una descripción incompleta de la gravedad de la Luna sobre la Tierra y las mareas (figura 1): «La capa fluida... que recubre la Tierra en los puntos más próximos a la Luna está sometida a una fuerza gravitatoria mayor (zona A), mientras que en los puntos más alejados (zona B) la fuerza es menor. De modo que existe una variación de la intensidad de la fuerza gravitatoria a lo largo del diámetro terrestre $\mathrm{AB}$, que tiende a estirar la capa fluida de forma parecida a un muelle. Por otro lado, la acción de las fuerzas gravitatorias en las zonas de la capa fluida perpendiculares al eje de la Tierra-Luna (puntos $\mathrm{C}$ y D) tiende a comprimirla». Es decir, la fuerza de atracción Tierra-Luna $\left(\mathrm{F}_{\mathrm{TL}}\right)$ en $\mathrm{B}$ va hacia adentro, como sucede con una de sus componentes en $\mathrm{D}$ y $\mathrm{C}$, y sin embargo en $\mathrm{D}$ y $\mathrm{C}$ produce marea baja y en $\mathrm{B}$ marea alta. 


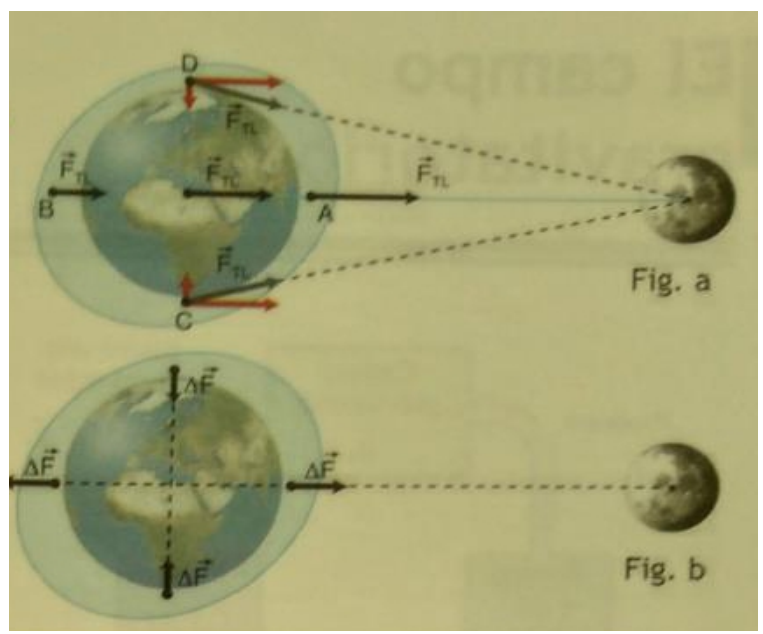

Figura 1. Figura explicativa de las mareas extraída de Lorente et al. 2009.

En la figura 1 aparecen unas diferencias de fuerzas $(\Delta \mathrm{F})$ con los sentidos correctos, pero con una explicación incorrecta, tal y como veremos en el siguiente apartado: «En el punto más próximo a la Luna la diferencia de la fuerza gravitatoria es mayor respecto al centro y por ello es positiva y dirigida hacia la Luna, y como la fuerza media debe ser constante, la diferencia en el otro extremo debe ser negativa, y dirigida en el sentido opuesto al lado contrario».

Dalmau, Pérez, Satoca y Tejerina (2009) afirman: «Una molécula de agua situada en A se ve más atraída por la Luna que el centro de la Tierra... Por el contario en B la atracción de la Luna es menor que en el centro de la Tierra y, por tanto, ese punto se verá menos atraído. Ahora es el centro de la Tierra el que cae hacia la Luna, y la molécula B se retrasa». Explicación plausible ya que compara A y B con el centro de masas (CM) de la Tierra.

Andrés, Antón y Barrio (2009), al ser conscientes de que la fuerza en B hacia el centro de la Tierra no puede explicar la marea alta en ese punto, introducen la fuerza centrífuga (Fc). Concretamente dicen: «La inercia hace que al girar un balón mojado las partículas de agua se desprendan de su superficie. De igual forma el movimiento de rotación de la Tierra provoca que el agua de los océanos tienda a salir despedida, pero no lo consigue por la atracción gravitatoria de la propia Tierra». En consecuencia, «la combinación de los dos fenómenos (la diferencia de la atracción y la fuerza centrífuga) hace que las regiones oceánicas situadas en la cara de la Tierra orientada hacia la Luna se acerquen hacia el satélite, por lo que se encuentran en pleamar. A la vez, las regiones en la cara opuesta de la Tierra se alejan del satélite y también se encuentran en pleamar».

Igualmente, en Vidal y Sánchez (2016) encontramos: «Este movimiento circular da lugar a que, por inercia, las masas de agua tiendan a moverse hacia el exterior de la órbita. A este movimiento centrífugo se suma la fuerza de atracción gravitatoria de la Luna, que es mucho más intensa en el lado más próximo de la Tierra que en el más alejado». Las Fc son fuerzas ficticias, que sólo aparecen en sistemas de referencia acelerados. La Fc es mayor en el ecuador y explica el achatamiento de la Tierra por los polos, y que el nivel del mar sea mayor en el ecuador que en las zonas templadas y, sobre todo, en las polares. La mayor altura de las aguas ecuatoriales por la Fc impide que las mareas sean claramente apreciables en el ecuador, ya que esa Fc se ejerce por igual en toda la circunferencia ecuatorial mientras que las mareas sólo aumentan ese nivel en el punto más cerca de la Luna y en el opuesto, y es un aumento de nivel mucho menor. 
En resumen, de los 17 libros de Física de Bachillerato analizados sólo cuatro tratan las mareas, dos utilizando erróneamente fuerzas centrífugas, otro hablando de supuestas caídas y el cuarto introduce un $\Delta \mathrm{F}$ hacia fuera en $\mathrm{B}$, pero con una explicación incorrecta.

El análisis de manuales de enseñanza superior muestra que en los cursos de ciencia e ingeniería este tema es muy poco tratado en la enseñanza de la Física. Esta situación no ha variado en el transcurso del tiempo, como se comprueba por la muestra que describimos a continuación y que contiene manuales editados desde 1971 hasta 2009. Empezamos con libros estadounidenses de Física, de uso común en el resto del mundo, muchos de los cuales se siguen reeditando en la actualidad. De esta manera, desde la edición analizada más reciente a la más antigua encontramos que:

- En Sears, Zemansky, Young y Freedman (2009) sólo se calcula la aceleración de la gravedad en la superficie de la Luna, viendo que es más pequeña que la terrestre, lo que explica el poco peso de los astronautas en la Luna. Curiosamente no se abordan las mareas en la Tierra, pero sí se habla cualitativamente de las mismas en los agujeros negros.

- En Halliday, Resnick y Krane (2007) apenas se habla de las mareas en el cuerpo teórico del libro (salvo en el apartado "patrón de tiempo" para mostrar cómo estas alteran la duración del día). En el tema de gravitación se compara la gravedad de la Tierra sobre la Luna y sobre una manzana y hay varias preguntas en las que se plantea por qué si la atracción directa del Sol sobre la Tierra es 175 veces mayor que la de la Luna, sin embargo, su efecto sobre las mareas es de la mitad respecto al de la Luna o por qué tanto en Luna llena como nueva se suman los efectos gravitacionales del Sol y la Luna. En un tema posterior se pide una expresión para la fuerza ejercida por la Luna sobre una partícula de agua situada en la Tierra en un punto A ubicado bajo la Luna. A continuación, supone que la partícula de agua está en el centro de la Tierra y se pregunta la fuerza que ejercería la Luna sobre ella y después que se calcule la diferencia de estas fuerzas, que representa la fuerza de mareas, que es la fuerza sobre el agua con relación a la Tierra. Luego pide que se repita el cálculo para una partícula de agua situada en un punto B localizado en el lado más lejano de la Tierra respecto a la Luna. $\mathrm{Si}$ bien en el problema se plantean las preguntas correctas, es muy difícil que el alumnado pueda contestarlas sin la guía del profesorado o sin conocer las aproximaciones que necesita realizar, que no se sugieren. Por otra parte, no se explica por qué hay que calcular la diferencia entre la fuerza de la gravedad en el centro de la Tierra y en la superficie. Y, por último, al aparecer en el apartado de preguntas y cuestiones, es decir, fuera del cuerpo central del tema, el profesorado puede decidir no tratarlas.

- En Hewit (2004), un curso introductorio a la Física, en el tema de gravitación dedica cuatro páginas a las mareas (es el libro de Física que más espacio les dedica), aunque realizando un tratamiento cualitativo acorde con el resto del libro. Compara las mareas producidas por el Sol y por la Luna en la Tierra, mostrando que la del primero es menor porque varía con la inversa del cubo de la distancia $\left(1 / r^{3}\right)$ a la Tierra. Muestra, sin calcularlo, que las personas no tienen altura suficiente para que la Luna produzca mareas en ellas, ya que su efecto sería 0,002 veces menor que el producido por una calabaza de $1 \mathrm{~kg}$ a $1 \mathrm{~m}$ de la cabeza de la persona. Introduce que las mareas son vivas y muertas cuando se alinean Sol, Tierra y Luna y menciona las mareas en la corteza terrestre y la atmósfera. Y, con cierto estilo humorístico, dice: «¿Has notado que 
alguno de tus amigos parece más raro en Luna llena?», texto que pone de manifiesto las creencias populares sobre comportamientos anómalos en Luna llena. Sin embargo, este comentario es un tanto contradictorio, ya que si tuviese causas gravitatorias se produciría el mismo efecto sobre el comportamiento en Luna nueva.

- En Alonso y Finn (1995) se habla de nuestro satélite en el apartado "Viaje a la Luna" del capítulo de exploración en el espacio. No trata ni mareas ni iluminancia.

- En Tipler (1992) solo se aborda el cálculo de la aceleración de la gravedad (o campo gravitatorio) de la Tierra sobre la Luna y no se tratan las mareas.

- En Gettys, Keller y Skove (1991) se calcula el punto donde la aceleración de la gravedad terrestre coincide con la de la Luna. En un texto complementario se abordan las mareas en la bahía de Fundy (Canadá), que alcanzan una amplitud mareal de hasta $15 \mathrm{~m}$, cuando en su entorno son de unos $3 \mathrm{~m}$. Esto lo atribuyen a la resonancia y por eso el texto aparece en el tema de oscilaciones.

- En Giancoli (1985) no hay un tema de gravitación propiamente dicho, sino que esta se incluye en el tema de movimiento circular y de rotación. Las mareas solo se mencionan en el tema de inducción electromagnética, en un párrafo sobre energía maremotriz.

- En Holton y Brush (1976), un manual introductorio de Física con un enfoque histórico desarrollado en la Universidad de Harvard, encontramos dos párrafos con consideraciones cualitativas: que la marea se produce poco después del paso de la Luna por el meridiano del lugar y $12 \mathrm{~h}$ después, y que dos veces al mes, en Luna llena y nueva, estas mareas son vivas y muertas, al sumarse la influencia del Sol. Así mismo, señalan que las características del fenómeno dependen de la topografía de la costa y del fondo del océano.

- En Feynman, Leighton y Sands (1971), uno de los referentes para la enseñanza de la Física, más utilizado por el profesorado que por el alumnado, encontramos una breve explicación cualitativa de dos párrafos sobre las mareas, usando la fuerza centrífuga, en el apartado de la Ley de gravitación de Newton. También incluye aspectos de iluminación como la Ley de Lambert $\left(\mathrm{E}=\mathrm{I} / \mathrm{r}^{2}\right.$, donde $\mathrm{E}$ es el nivel de iluminación en lux, I es la intensidad de la fuente en candelas y $\mathrm{r}$ es la distancia de la fuente de luz al plano receptor perpendicular, en metros).

En relación con el análisis de libros de Física de otros países:

- El texto alemán de Gerthsen, Kneser y Vogel (1979) es uno de los pocos que trata todos los temas, aunque muy brevemente. Calcula la gravedad de la Tierra sobre la Luna e introduce una reducida y poco clarificadora explicación sobre la aceleración de las mareas $\left(a_{m}\right)$, mediante la expresión $a_{m}=-2 G m R / r^{3}$ (siendo $G$ la constante de gravitación universal, $\mathrm{R}$ el radio de la Tierra, $\mathrm{m}$ la masa de la Luna, y $\mathrm{r}$ la distancia Tierra-Luna), que aproxima a $\mathrm{a}_{\mathrm{m}}=10^{-7} \mathrm{~g}$. Introduce medio capítulo de fotometría, donde trata flujo, luminosidad e iluminancia, aunque la denomina densidad de iluminación.

- El texto ruso de Yavorski y Detlaf (1977) introduce la Ley de gravitación universal (con solo la particularidad de la gravedad en la superficie terrestre), así como definiciones y unidades fotométricas, algunas con denominaciones no habituales.

Por último, presentamos el análisis de dos textos españoles: 
- Carreras, Fernández-Rañada, García y Yuste (1993) introducen la luminosidad del Sol $\left(3,79 \cdot 10^{26} \mathrm{~J} / \mathrm{s}\right)$, sin compararla con la de la Luna. Calculan la gravedad de la Tierra sobre la Luna. Para las mareas calculan adecuadamente, utilizando la aproximación de Taylor, la gravedad (g) en el punto de la Tierra más próximo (A) $g_{\mathrm{A}}$ y más lejano (B) $\mathrm{g}_{\mathrm{B}}$ a la Luna, encontrando que en $\mathrm{B}$ va dirigida hacia el centro de la Tierra, lo que debería producir una marea baja, al contrario de lo que sucede (ver figura 5). Para explicarlo enuncian la siguiente frase: «el diámetro $\mathrm{AB}$ tenderá a estirarse (de la misma forma que se estira un muelle sobre cuyos extremos actúan fuerzas diferentes)». En Hewitt (2004) también aparece una expresión similar: «Una esfera de gelatina permanece esférica cuando se tira de todas sus partes por igual en la misma dirección. Sin embargo, cuando uno de sus lados es atraído más que el otro se alarga». Ambos modelos o analogías son sólo una primera aproximación porque si se aplican fuerzas distintas en el mismo sentido, es cierto que el muelle o la gelatina se deforman, pero no como lo hacen las mareas, ya que donde se aplica la fuerza hacia adentro la deformación se produce hacia adentro.

- En Ortuño (1996), un libro de Física para Biología, Medicina, Veterinaria y Farmacia, no se trata ni gravitación ni mareas y tan solo se dedica un breve apartado de Fotometría para introducir flujo, intensidad e iluminancia.

Con la finalidad de encontrar más información sobre las mareas, al ser tan escasa en los libros de Física (en especial en los más cuantitativos), hemos analizado dos libros de Astronomía:

- En Bakulin, Kononovich y Morov (1987) se calcula la aceleración de la Tierra sobre la Luna. No se calcula la aceleración de la gravedad en el punto de la Tierra más próximo $\mathrm{g}_{\mathrm{A}}$ y más lejano $\mathrm{g}_{\mathrm{B}}$ a la Luna, pero sí se calcula la aceleración relativa en $\mathrm{A}\left(\mathrm{gr}_{\mathrm{A}}\right)$ respecto al CM, utilizando para ello una aproximación ad hoc. El desarrollo completo es el siguiente:

$g_{r A}=g_{A}-g_{L}=G \frac{m}{(r-R)^{2}}-G \frac{m}{r^{2}}=G \cdot m \frac{\left(r^{2}-(r-R)^{2}\right)}{(r-R)^{2} \cdot r^{2}}=G \cdot m \frac{2 r R-R^{2}}{(r-R)^{2} \cdot r^{2}}=\left(\begin{array}{c}C o m o R \ll r \\ 2 r R-R^{2} \approx 2 R r \\ (r-R)^{2} \approx r^{2}\end{array}\right) \simeq G \cdot m \frac{2 r R}{r^{4}}=\frac{2 G m R}{r^{3}}$

Siendo $g_{\mathrm{L}}$ el efecto de gravedad en el CM de la Tierra producida por la Luna. Del mismo modo, se calcula la aceleración relativa en $\mathrm{B}\left(\mathrm{gr}_{\mathrm{B}}\right)$ que resulta ser igual y de sentido contrario, por lo que ambas van hacia afuera de la superficie terrestre, produciendo mareas altas. Se dibuja, aunque sin calcularlas, las aceleraciones relativas en $\mathrm{C}$ y D ( $\left(\mathrm{r}_{\mathrm{C}} \mathrm{y} \mathrm{gr}_{\mathrm{D}}\right.$, respectivamente), que van hacia dentro de la Tierra, produciendo mareas bajas. También se calcula la marea creada por el Sol, que es 2,2 veces menor que la de la Luna y se indica que «durante los novilunios y plenilunios los flujos solar y lunar comienzan simultáneamente, las acciones de la Luna y del Sol se suman y se observa la marea máxima de flujo». Después se dedica un capítulo, el quinto, a los movimientos de la Luna, un apartado a la magnitud estelar absoluta y luminosidad de las estrellas, y otro a la constante solar (la energía de la radiación solar por unidad de tiempo y por unidad de superficie), que es de $1.360 \mathrm{~W} / \mathrm{m}$.

- Martínez, Miralles, Marco y Galadí-Enríquez (2005) dedican un apartado a la Luna, en el que se describe sus movimientos (rotación y libración y eclipses de Sol y de Luna), y otro a las mareas en el que se calculan $\mathrm{gr}_{\mathrm{A}}$ y $\mathrm{gr}_{\mathrm{B}}$ respecto al $\mathrm{CM}$, utilizando la misma aproximación ad hoc que Bakulin et al. (1987), al que citan en la bibliografía básica del libro. Indican que las mareas producen un rozamiento que ha ido alargando la duración del día, ya que hace 500 millones de años era de $22 \mathrm{~h}$. Como el momento 
angular del sistema Tierra-Luna permanece constante, esto hace que el de la Luna aumente $\mathrm{y}$, en consecuencia, se aleja $4 \mathrm{~cm} /$ año. Introducen la luminosidad del Sol $\left(3,85 \cdot 10^{26} \mathrm{~J} / \mathrm{s}\right)$ e indican que la de las estrellas varía entre $10^{-4}$ y $10^{6}$ veces respecto a la del Sol.

Para encontrar más información sobre la luminosidad, al ser esta escasa en los libros de Física, se han analizado dos textos de Óptica: Smith y Thomson (1989) y Casas (1985). En el capítulo de Fotometría, ambos introducen las principales magnitudes como flujo, intensidad e iluminancia, pero sin ninguna aplicación a la Luna.

En resumen, encontramos que los libros de Física, incluso los que estudian aplicaciones de la física en biología (Giancoli 1985, Ortuño 1996), tampoco se ocupan de la influencia de la Luna en los seres vivos, ni en las mareas. Estas sólo se tratan en algunos libros que presentan las ideas básicas de la física en un lenguaje sin tecnicismos y con uso mínimo de las matemáticas (Carreras et al. 1993; Hewitt 2004) o, con mayor rigurosidad, en libros de astronomía (Bakulin et al. 1987; Martínez et al. 2005). Hay una tendencia a la desaparición de los temas considerados de ciencia aplicada, como el caso de las mareas, pese a su gran relevancia económica y social, ignorando su importancia en la educación Ciencia-TecnologíaSociedad y su contribución al pensamiento crítico (Torres y Solbes, 2016).

Ante este tratamiento en los manuales tan mejorable, los estudiantes de Bachillerato y universitarios, que mayoritariamente saben que el fenómeno de las mareas está relacionado con la atracción gravitatoria de la Luna sobre nuestro planeta, tienen en su mayor parte dificultades a la hora de representar un modelo a escala global que explique el origen del fenómeno (Corrochano, Gómez-Gonçalves, Sevilla y Pampín-García 2017; Viiri 2000). Por eso, no resulta sorprendente la permanencia de las creencias pseudocientíficas en la ciudadanía y, de manera más preocupante, en el futuro profesorado de materias científicas. Tal y como mostraban Solbes, Palomar y Domínguez-Sales (2018), el 64,9\% de una muestra de 131 estudiantes del Máster de Profesorado de Secundaria de la especialidad de ciencias, está de acuerdo o parcialmente de acuerdo con la expresión «La fase de la Luna puede afectar en cierta medida cuestiones como la salud de las personas, el nacimiento de niños o ciertos trabajos agrícolas».

\section{La física de la Luna: una propuesta didáctica}

Aunque existen propuestas didácticas para introducir de manera cualitativa las mareas en niveles más básicos (Armario, Oliva y Jiménez-Tenorio 2019), en este apartado mostramos una secuencia de actividades (SEA) para introducir cuantitativamente aspectos sobre la física de la Luna que consideramos necesarios para poner en cuestión las supuestas influencias lunares en los seres vivos con alumnado de Física en $2^{\circ}$ de Bachillerato o en $1^{\circ}$ de Universidad, en la unidad didáctica de Gravitación. Para la SEA completa se prevé unas 3-4 h de duración, pero también se propone una selección de actividades, evitando los cálculos más complejos, que se podría implementar en $2 \mathrm{~h}$. Se trata de actividades reflexivas y abiertas a realizar en grupos de tres o cuatro personas, que favorecen el nivel de participación y la creatividad necesarias, seguidas de puestas en común (para no emplear excesivo tiempo, un grupo presenta sus resultados y el resto los comentan) y de intervenciones del profesorado para realizar reformulaciones globalizadoras de las aportaciones de los grupos o para añadir información. En la tabla 2 se muestran los objetivos de las actividades. 
Tabla 2. Secuencia de aprendizaje en la que se indican las actividades y sus objetivos.

\begin{tabular}{|l|l|}
\hline Actividad & Objetivo \\
\hline A1 & Explicitar los conocimientos y creencias del alumnado. \\
\hline A2 & $\begin{array}{l}\text { Recopilar información sobre la Luna que utilizará el alumnado en posteriores actividades para } \\
\text { contrastar sus ideas previas con las evidencias científicas. }\end{array}$ \\
\hline A3 & $\begin{array}{l}\text { Determinar el campo gravitatorio de la Luna en la Tierra y reflexionar sobre el ínfimo valor } \\
\text { obtenido. }\end{array}$ \\
\hline A4 & Representar gráficamente dicho campo gravitatorio en los puntos de interés. \\
\hline A5 & $\begin{array}{l}\text { Representar gráficamente las aceleraciones relativas al centro de la Tierra en los mismos } \\
\text { puntos de la A4, comprobando que gra va hacia fuera. }\end{array}$ \\
\hline A6 & Calcular las aceleraciones en los puntos A y B mediante aproximación matemática adecuada. \\
\hline A7 & $\begin{array}{l}\text { Calcular las aceleraciones relativas respecto al CM en dichos puntos y su relación con las } \\
\text { mareas altas. }\end{array}$ \\
\hline A8 & Calcular la aceleración relativa en los puntos C y D y ru relación con las mareas bajas. \\
\hline A9 & $\begin{array}{l}\text { Buscar información sobre mareas vivas y muertas y su relación con las posiciones de la Luna y } \\
\text { el Sol. }\end{array}$ \\
\hline A10 & $\begin{array}{l}\text { Calcular el efecto de la marea sobre los extremos de un ser vivo y comprobar que es menor } \\
\text { que el producido por una masa de 1 kg a 1 m de distancia. }\end{array}$ \\
\hline A11 & Reflexionar por qué las mareas en el Mediterráneo son inapreciables. \\
\hline A12 & Obtener conclusiones mediante la comparación de las iluminancias del Sol y la Luna. \\
\hline
\end{tabular}

A continuación, se incluye la SEA completa mostrando el enunciado de cada actividad en cursiva y los comentarios e información sobre la misma en letra redonda.

\section{A.1. ¿Sobre qué fenómenos cotidianos crees que influye la Luna?}

Es habitual que mencionen los que ya hemos señalado en la introducción: en los nacimientos, en el aumento de la criminalidad y de la violencia, en las tasas de suicidios e ingresos hospitalarios, efectos psiquiátricos, aumento del consumo de alcohol y otras drogas, en el sonambulismo, en la menstruación, en la agricultura (en el crecimiento de las plantas, en periodos de siembra, de poda...), etc. Esto sucede porque forman parte del imaginario popular.

\section{A.2. Busca información sobre las características astronómicas de la Luna}

La información básica que pueden encontrar puede ser la siguiente: La Luna es el único satélite natural de nuestro planeta y describe a su alrededor una órbita elíptica con un semieje mayor (a) de $384.000 \mathrm{~km}$, con una excentricidad (e) de 0,0549 y con un ángulo de $5^{\circ}$ 9' con respecto al plano de la eclíptica, lo que explica que no se produzcan eclipses de Sol o de Luna cada Luna nueva o Luna llena. El tiempo que tarda en completar una órbita alrededor de la Tierra con respecto a las estrellas fijas es de 27,3 días (mes sidéreo), mientras que tarda 27,2 días en pasar entre dos posiciones consecutivas de paso en un mismo nodo (mes draconítico). En cambio, tarda 29,5 días en orbitar alrededor de la Tierra y volver a su posición análoga con respecto al Sol y la Tierra (ciclo o mes lunar, también conocido como mes sinódico) (figura 2). La diferencia entre el mes sidéreo y sinódico se debe al hecho de que la Luna se mueve junto a la Tierra alrededor del Sol (Martínez et al. 2005). Sin embargo, un sitio específico de la Tierra tarda en rotar desde un punto exacto bajo la Luna hasta el mismo punto 24 horas y 50 minutos (día lunar) (Morgan 2001). La acción combinada de estos dos ciclos (mes y día 
lunares) tienen diferentes efectos en la Tierra, como la variación de su iluminación y las mareas.

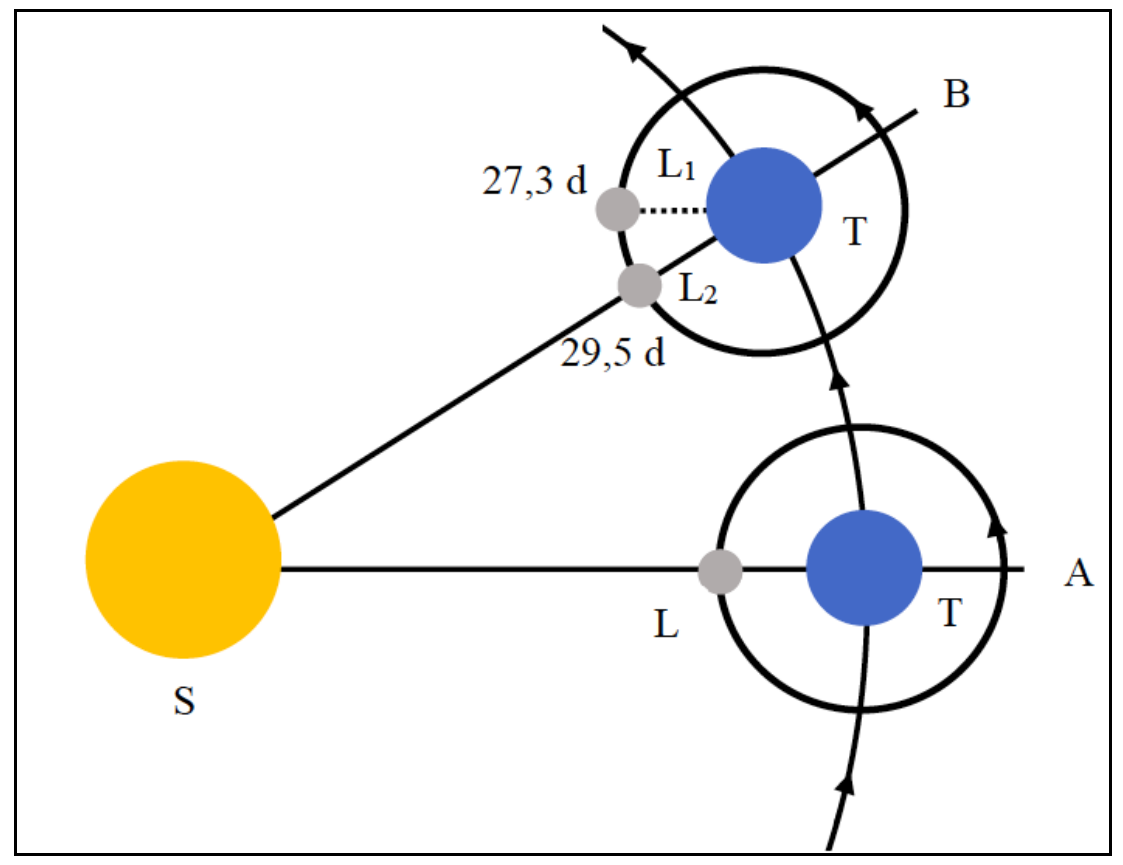

Figura 2. Diagrama para ilustrar los cambios en la configuración del Sol (S)-Tierra (T)-Luna (L) durante un mes lunar. En el momento A, la Luna se encuentra entre el Sol y la Tierra (Luna nueva). En el momento B, la Luna habrá regresado al mismo punto en relación con la Tierra 27,3 días o un mes sideral más tarde $\left(\mathrm{L}_{1}\right)$. Sin embargo, la Luna necesitará otros 2,2 días, es decir, un total de 29,5 días o un mes sinódico $\left(\mathrm{L}_{2}\right)$, para volver a su posición en la línea entre la Tierra y el Sol (redibujado a partir de Rackham 1968). No está a escala.

\section{A.3. Determina el campo gravitatorio (o aceleración) de la Luna en el CM de la Tierra $\left(g_{L}\right)$.}

Desde el punto de vista gravitacional, el efecto de la gravedad en el CM de la Tierra producida por la Luna $\left(g_{\mathrm{L}}\right)$ se puede calcular mediante la expresión $\mathrm{g}_{\mathrm{L}}=\mathrm{Gm} / \mathrm{r}^{2}$, siendo $\mathrm{G}$ la constante de gravitación universal, $\mathrm{m}$ la masa de la Luna y r la distancia Tierra-Luna (T-L). Como $\mathrm{m}=\mathrm{M} / 80$ y r $=60 \mathrm{R}$ (donde $M$ es la masa de la Tierra y $\mathrm{R}$ su radio), y por tanto $\mathrm{g}=\mathrm{GM} / \mathrm{R}^{2}=9,8 \mathrm{~ms}^{-2}$, el valor de la aceleración de la gravedad de la Luna en la superficie terrestre $\left(\mathrm{g}_{\mathrm{L}}\right)$ es:

$$
g_{L}=G \frac{m}{r^{2}}=G \frac{\frac{M}{80}}{(60 R)^{2}}=G \frac{M}{\left(80 \cdot 60^{2}\right) \cdot R^{2}}=\frac{9,8}{288000} \approx 3 \cdot 10^{-5} \mathrm{~ms}^{-2}
$$

es decir, el valor de dicha gravedad es 288.000 veces menor que la gravedad terrestre. Se trata pues, desde el punto de vista gravitatorio, de un efecto despreciable. Incluso la gravedad del Sol $\left(g_{\mathrm{s}}\right)$ sobre la Tierra es 177 veces superior a la de la Luna $\left(g_{\mathrm{s}}=\mathrm{g} / 1,627=177 \mathrm{~g}_{\mathrm{L}}=6 \cdot 10^{-3} \mathrm{~ms}^{-2}\right)$. 
A.4. Representa gráficamente en la figura 3 adjunta los campos gravitatorios (o aceleraciones) que ejerce la Luna (L) en el CM y sobre distintos puntos de la superficie terrestre $A, B, C$ y $D$.

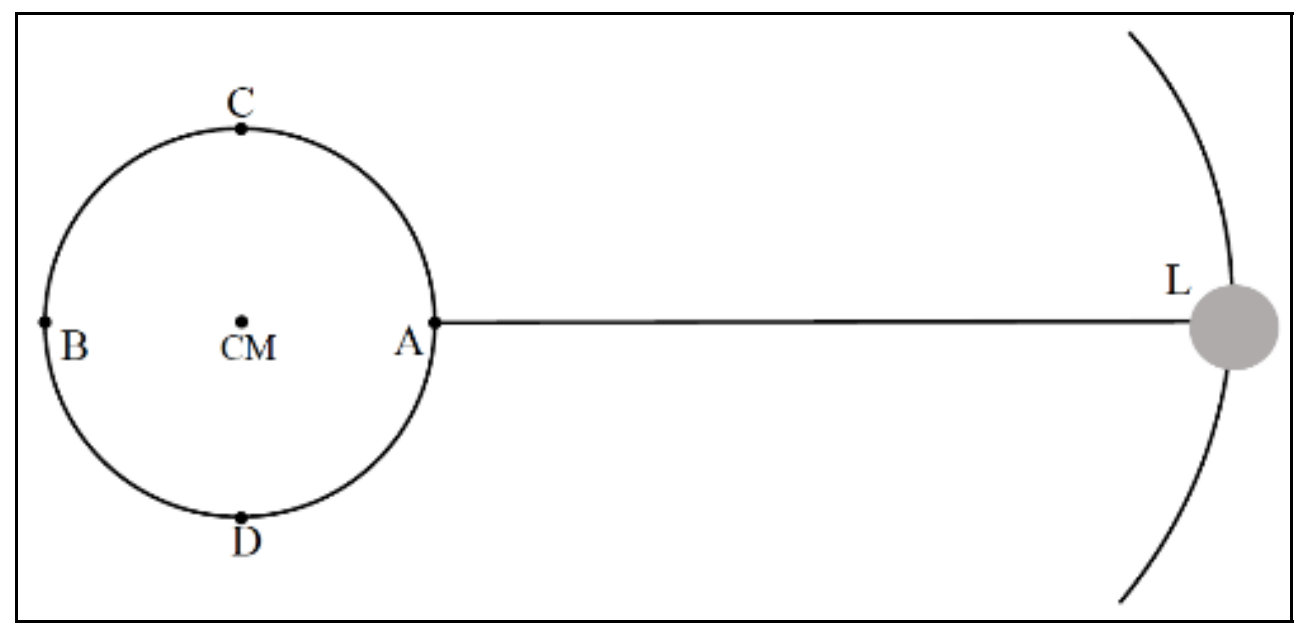

Figura 3. Representación de los puntos CM, A, B, C y D en los que se calculan los campos gravitatorios. Fuente: Elaboración propia.

Los campos gravitatorios (o aceleraciones) que ejerce la Luna en el CM de la Tierra y sobre distintos puntos de la superficie terrestre A, B, C y D tienen su origen en la Luna. Pero sólo $g_{A}$ tiene sentido hacia fuera, por tanto, sólo ella puede producir mareas altas (figura 4).

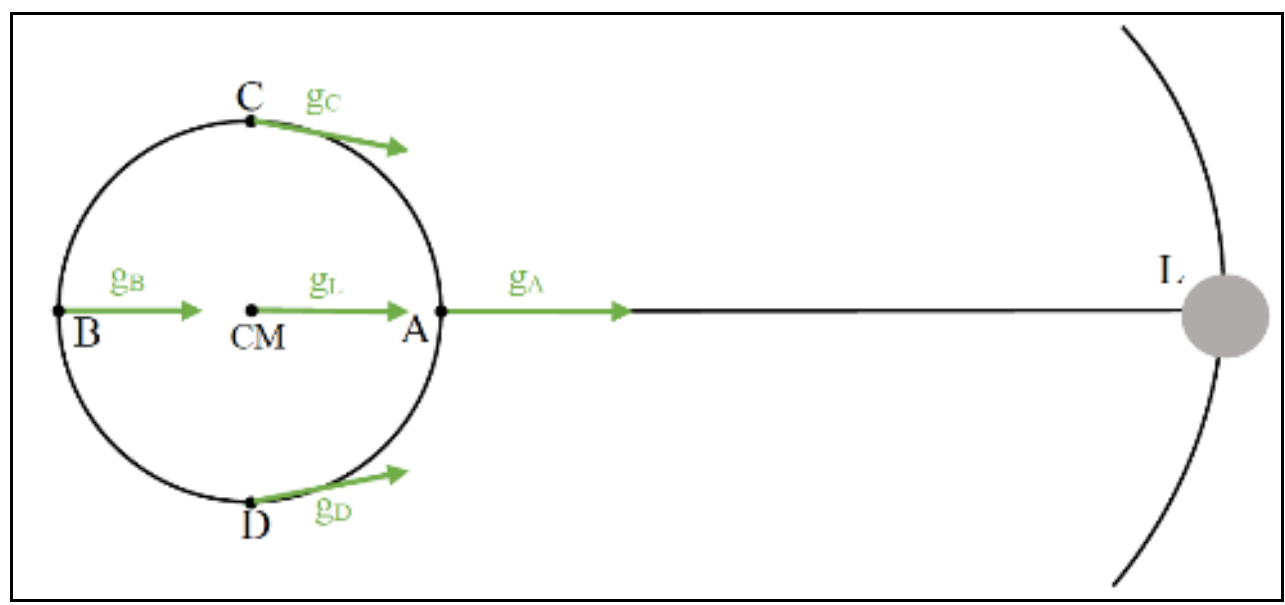

Figura 4. Representación de los campos gravitatorios en el CM, A, B, C y D. Fuente: Elaboración propia.

A.5. Las aceleraciones anteriores se han calculado respecto al centro de la Luna, pero como las mareas son fenómenos en la Tierra, hay que calcular esas aceleraciones respecto al centro de la Tierra. Para ello hay que restar la gravedad de la Luna en el centro de la Tierra. Es como cuando vamos en un barco: respecto a la costa estamos en movimiento, pero el barco respecto a nosotros está en reposo. Por tanto, para calcular la velocidad (o aceleración) en cualquier punto de la nave hay que restarle su velocidad (o la aceleración) respecto a la costa. Dibuja las aceleraciones relativas al centro de la Tierra en $A, B, C$ y $D$ denominadas respectivamente $\mathrm{gr}_{\mathrm{A}}$, $\operatorname{gr}_{\mathrm{B}}, \operatorname{gr}_{\mathcal{C}} y \mathrm{gr}_{\mathrm{D}}$.

Si queremos calcular la aceleración relativa respecto al CM de la Tierra en cualquiera de dichos puntos tenemos que restar la aceleración del CM gL. Por ejemplo, en $\mathrm{A}, \mathrm{gr}_{\mathrm{A}}=\mathrm{g}_{\mathrm{A}}-\mathrm{g}_{\mathrm{L}}$ y así en el resto de los puntos (figura 5). Mediante cálculos sencillos, que veremos en siguientes actividades, comprobaremos que $\mathrm{gr}_{\mathrm{A}}=\mathrm{gr}_{\mathrm{B}}=\mathrm{g}_{\mathrm{L}} / 30$, pero ambas $\left(\mathrm{gr}_{\mathrm{A}} \mathrm{y} \mathrm{gr}_{\mathrm{B}}\right)$ dirigidas hacia fuera, lo que puede explicar que en dichos puntos exista marea alta. 


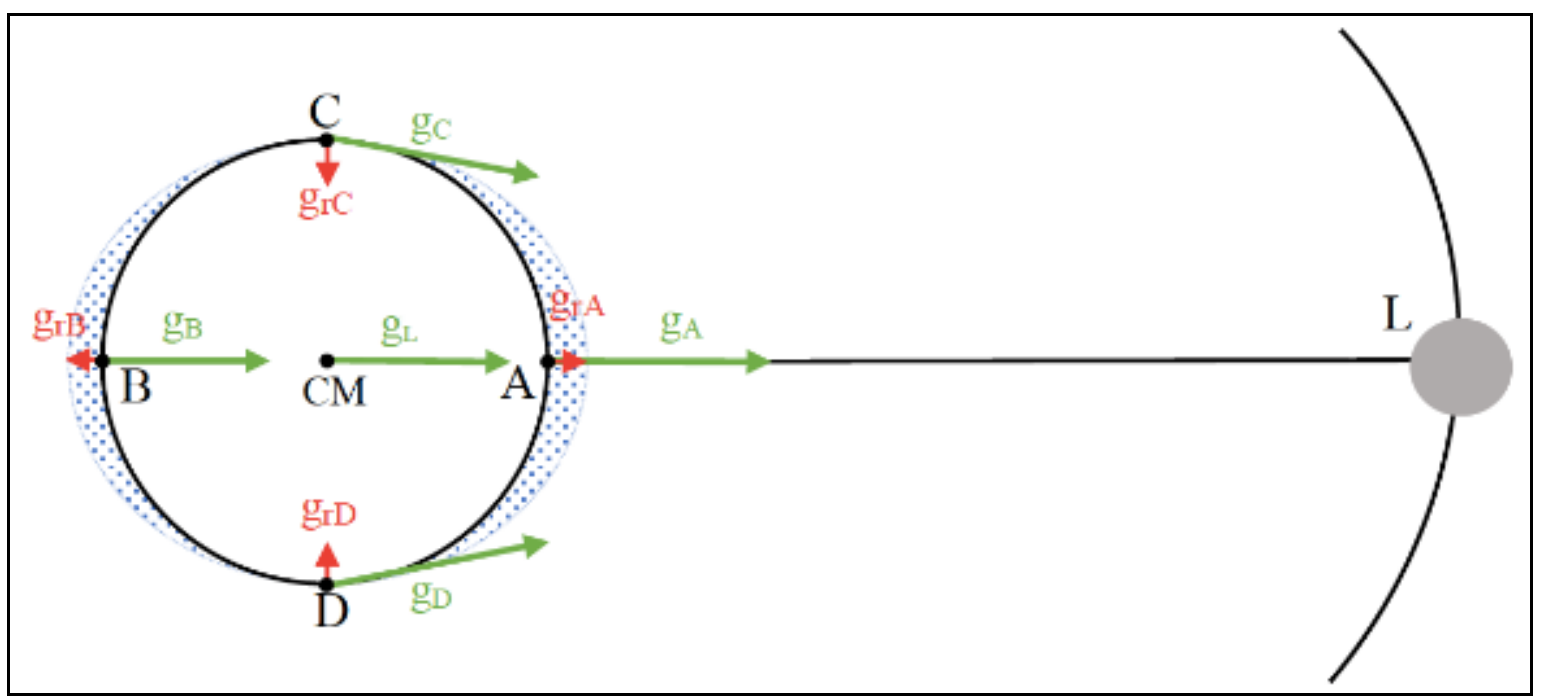

Figura 5. Representación de $\mathrm{g}_{\mathrm{L}}$, la aceleración en el CM de la Tierra causada por la atracción lunar; $\operatorname{gr}_{\mathrm{A}}$ y $\operatorname{gr}_{\mathrm{B}}$ son, respectivamente, las aceleraciones de los puntos A y B relativas al CM de la Tierra, situados en extremos opuestos de la superficie terrestre sobre la línea Tierra-Luna y gr es la aceleración relativa al CM terrestre. Modificado de Bakulin et al. (1987).

A.6. Calcula $g_{A}$ y $g_{B}$ utilizando la aproximación en serie $\left[1 /(1-x)^{2} \simeq 1+2 x+3 x^{2} \ldots \simeq 1+2 x\right]$ que es válida si $x$ es pequeña como en el caso $x=R / r$, siendo $r$ la distancia Tierra-Luna y $R$ el radio de la Tierra, $R=r / 60$.

Aunque no se conozca la aproximación, la validez de esta se puede comprobar para $\mathrm{x}$ pequeñas. Es recomendable usarla, porque simplifica notablemente todos los cálculos. Para $g_{A}$ sería:

$$
g_{A}=G \frac{m}{(r-R)^{2}}=G \frac{m}{r^{2} \cdot\left(1-\frac{R}{r}\right)^{2}} \simeq \frac{G m}{r^{2}}\left(1+\frac{2 R}{r}\right)
$$

Igualmente, en g $\mathrm{B}_{\mathrm{B}}$ sería:

$$
g_{B}=G \frac{m}{(r+R)^{2}}=G \frac{m}{r^{2} \cdot\left(1+\frac{R}{r}\right)^{2}} \simeq \frac{G m}{r^{2}}\left(1-\frac{2 R}{r}\right)
$$

En resumen, en función de la gravedad de la Luna, $g_{L}=G \frac{m}{r^{2}}$ tenemos que:

$$
\begin{aligned}
& g_{A} \simeq \frac{G m}{r^{2}}\left(1+\frac{2 R}{r}\right)=g_{L}\left(1+\frac{2 R}{r}\right)=g_{L}\left(1+\frac{2 R}{60 R}\right)=g_{L}\left(1+\frac{1}{30}\right)=g_{L}\left(\frac{31}{30}\right) \\
& g_{B} \simeq \frac{G m}{r^{2}}\left(1-\frac{2 R}{r}\right)=g_{L}\left(1-\frac{2 R}{r}\right)=g_{L}\left(1-\frac{2 R}{60 R}\right)=g_{L}\left(1-\frac{1}{30}\right)=g_{L}\left(\frac{29}{30}\right)
\end{aligned}
$$

Es decir, se produce a lo largo del diámetro una variación del campo gravitatorio creado por la Luna. Un cuerpo situado en A experimenta una aceleración 1,03 veces mayor que en el CM y en B 0,97 veces mayor. Pero, evidentemente en B va hacia el centro de la Tierra, por ello se debería producir una marea baja, lo que no sucede. Por tanto, en la explicación de las mareas se debe tener en cuenta el valor de la gravedad relativa en el punto, que veremos a continuación. 
A.7. Determina las aceleraciones relativas en $A\left(g_{r A}=g_{A}-g_{L}\right)$ y $B\left(g_{r B}=g_{B}-g_{L}\right)$ respecto al CM.

La aproximación en serie utilizada por Carreras et al. (1993) en el cálculo anterior, pero no en este, simplifica los cálculos con respecto a la de Bakulin et al. (1987) vista anteriormente, de forma que:

$$
g_{r A}=g_{A}-g_{L} \simeq \frac{2 G m R}{r^{3}}=\frac{2 R g_{L}}{r}
$$

De la misma manera:

$$
g_{r B}=g_{B}-g_{L} \simeq \frac{-2 G m R}{r^{3}}=\frac{-2 R g_{L}}{r}
$$

Es decir, la aceleración relativa respecto al CM depende de la distancia entre A y B y de la distancia Tierra-Luna al cubo, en vez de al cuadrado. Substituyendo los valores numéricos, tenemos que el valor de $\mathrm{gr}_{\mathrm{B}}$ y $\mathrm{gr}_{\mathrm{A}}$ es de $\mathrm{g}_{\mathrm{L}} / 30=10^{-6} \mathrm{~ms}^{-2}$ siendo en los dos puntos igual, pero de sentido contrario: en el punto A va dirigido hacia la Luna y en el B en sentido contrario, ambas hacia fuera de la Tierra. Así, en A y en B se producirá marea alta a intervalos de aproximadamente 12,4 h, reflejando el día lunar de 24,8 h. Y debido a la rotación diaria de la Tierra, las mareas suben y bajan en la mayoría de las zonas costeras y estuarios de las grandes masas de agua dos veces cada día lunar.

Se puede añadir (o pedir al alumnado que busque información al respecto) que, en realidad, este modelo (que permite realizar cálculos exactos de forma bastante sencilla) también tiene limitaciones, porque el fenómeno es más complicado. La Tierra no está totalmente cubierta por océanos, por lo que la marea, en su desplazamiento por la superficie del océano, encuentra en su camino las líneas litorales de los continentes, las diferentes formas del fondo del mar y, en consecuencia, experimenta rozamiento. Por ello la marea no coincide con el momento de culminación de la Luna, sino que llega retrasada. Además, la amplitud de la marea no es la misma en los distintos lugares. En el océano, lejos de la costa, la amplitud no supera $1 \mathrm{~m}$, pero en el litoral, dependiendo de la orografía y profundidad del mar, la amplitud mareal puede alcanzar más de $10 \mathrm{~m}$, como en las bahías del Monte Saint-Michel (en el Atlántico Norte), de Frobisher (en el ártico canadiense) o la ya mencionada de Fundy (en la costa atlántica de Canadá), entre otras.

A.8. Explica lo que ocurre en los puntos $C y D$ de la figura 6, utilizando relaciones trigonométricas.

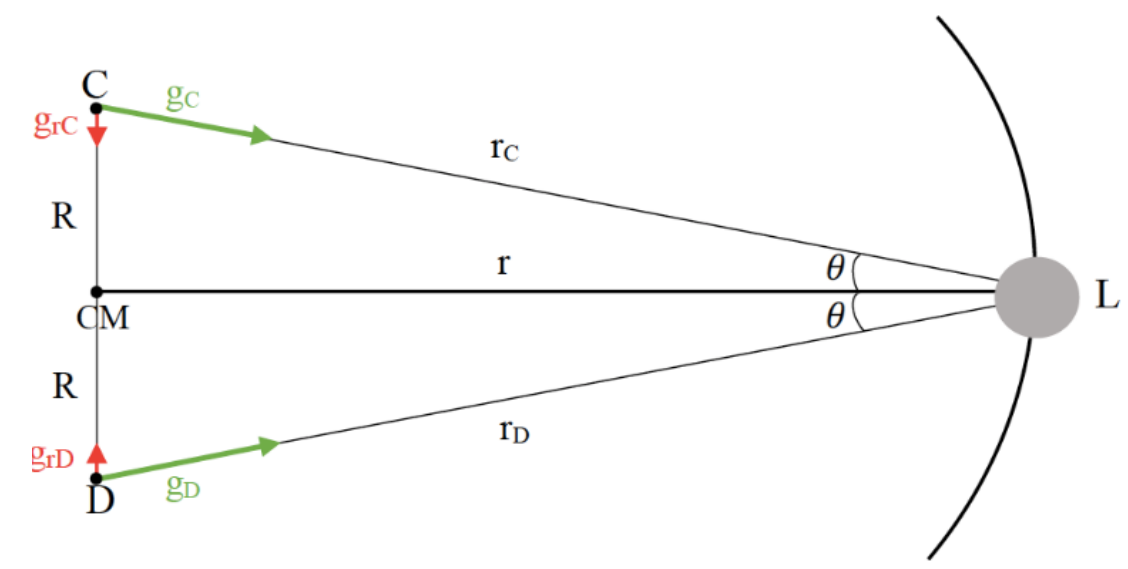

Figura 6. Representación de las aceleraciones relativas en C y D. Fuente: Elaboración propia. 
En el punto C, atendiendo a esta configuración:

$$
g_{C}=G \frac{m}{r_{C}^{2}}=G \frac{m}{\left(R^{2}+r^{2}\right)}
$$

La $g_{C}$ tiene una ligera inclinación respecto a la horizontal y, por tanto, su componente horizontal se anula con $g_{\mathrm{L}}$ y para calcular la vertical $\mathrm{g}_{\mathrm{rC}}$ hay que tener en cuenta que al $\operatorname{ser} \mathrm{R}<\mathrm{r}$,

entonces $\mathrm{R}^{2}+\mathrm{r}^{2} \simeq \mathrm{r}^{2}$, y utilizando el valor $g_{M}=G \frac{m}{r^{2}}$ tenemos que:

Es decir, su valor es $g_{\mathrm{L}} / 60$, la mitad que en $\mathrm{A}$ y $\mathrm{B}$, y con dirección y sentido hacia el centro de la Tierra, produciendo mareas bajas. Lo mismo sucede en D, en la parte opuesta de la Tierra.

\section{A.9. ¿Qué son las mareas vivas y muertas?}

Son el efecto combinado de la gravedad de la Luna y del Sol. Aunque la gravedad del Sol sobre la Tierra es mayor que la de la Luna, como en las mareas influye con la inversa de la distancia al cubo $\left(1 / \mathrm{r}^{3}\right)$, su efecto es menor que el de la Luna, al ser su distancia mucho mayor. En efecto, si tenemos en cuenta que la distancia Sol-Tierra es $\mathrm{r}=1 \mathrm{UA}=15 \cdot 10^{10} \mathrm{~m}$ y su masa es $\mathrm{m}=2 \cdot 10^{30} \mathrm{~kg}$, entonces su aceleración de marea vendrá dada por $2 \mathrm{GmR} / \mathrm{r}^{3}=5 \cdot 10^{-7} \mathrm{~ms}^{-2}$, que es 2,24 veces menor que la aceleración de marea lunar $\left(1,13 \cdot 10^{-6} \mathrm{~ms}^{-2}\right)$.

Así, cuando el Sol y la Luna están alineados con la Tierra (Luna llena y nueva), cada 14,77 días (medio mes sinódico: 29,53 días) es cuando se producen las mareas más altas, o mareas vivas, y cuando el eje que une el Sol con la Tierra y el eje que une la Luna con la Tierra forman un ángulo recto, es cuando se forman las mareas más bajas o mareas muertas (Morgan 2001). Es decir, la amplitud de las sucesivas mareas también es modulada durante un ciclo de 14-15 días, o semi-lunar. También se observan estas fuerzas de marea debido a la Luna y al Sol en la atmósfera y en la corteza terrestre (Adushkin, Riabova y Spivac 2017).

A.10. Calcula la diferencia entre el campo gravitatorio de la Luna sobre los extremos de un ser vivo de $2 m$ de altura (b) situado en la Tierra y compárala con la que produciría una masa de $1 \mathrm{~kg}$ a $1 \mathrm{~m}$ de distancia (r) sobre él.

El campo gravitatorio de la Luna sobre los extremos de un ser vivo de $2 \mathrm{~m}$ de altura situado en la Tierra es del orden de $2 \mathrm{~g}_{\mathrm{L}} \mathrm{h} / \mathrm{r}=3 \cdot 10^{-13} \mathrm{~ms}^{-2}$.

El campo gravitatorio que produciría una masa de $1 \mathrm{~kg}$ a $1 \mathrm{~m}$ de distancia sobre un ser vivo de $2 \mathrm{~m}$ de altura es $\mathrm{g}=\mathrm{Gm} / \mathrm{r}^{2}=6,67 \cdot 10^{-11} \cdot 1 / 1^{2}=6,67^{-11} \mathrm{~ms}^{-2}$ y por tanto un efecto marea del orden de $2 \mathrm{gh} / \mathrm{r}=6,67 \cdot 10^{-11} \cdot 4=2,67 \cdot 10^{-10} \mathrm{~ms}^{-2}$. Valores introducidos sin justificación por Hewitt (2004).

Por tanto, el primero es unas 1.000 veces menor que el segundo, con lo que el efecto de la Luna es tres órdenes de magnitud menor.

\section{A.11. ¿Por qué las mareas en el Mediterráneo son inapreciables?}

Como la marea depende del tamaño del objeto $2 \mathrm{~g}_{\mathrm{L}} \mathrm{h} / \mathrm{r}$, tenemos que en el Mediterráneo, al ser un mar casi cerrado, poco profundo y pequeño (con una profundidad media de $1.500 \mathrm{~m}$ ), las mareas son inapreciables. En cambio, en los océanos, al estar todos comunicados, podemos considerar que su tamaño es el del tamaño de la Tierra y, por tanto, las mareas son apreciables. 
A.12. ¿Existe influencia de la Luna en la fotosintesis que hacen las plantas? Cuando se habla de influencia de las fases lunares sobre el mundo vegetal, una de las creencias extendidas es que esta afecta a la fotosintesis debido a la variación de iluminancia. Define iluminancia, analiza la tabla de iluminancias que puedes encontrar en el enlace https://es.wikipedia.org/wiki/Lux, y compara la iluminancia de la Luna en sus diferentes fases con la del Sol.

La iluminancia es la cantidad de flujo luminoso que incide sobre una superficie por unidad de área. Su unidad en el Sistema Internacional (SI) es el lux: 1 lux $=1$ lumen $/ \mathrm{m}^{2}$. Cuando consulten la tabla del enlace, podrán observar que la iluminancia de la Luna varía de 0,001 lx en Luna nueva a 0,25 lx en Luna llena, pasando por los 0,01 lx de cuarto creciente o menguante.

Es conocido el papel de la luz en la fotosíntesis. Pero según la tabla de iluminancias, el mínimo de luz solar en un día medio es de 32.000 lx y el máximo de 100.000 lx, es decir, la iluminación máxima de la Luna llena en una noche clara es 128.000 veces menor que el mínimo de la luz solar en un día promedio, o 400.000 veces menor que el máximo. Nuevamente, el efecto de la Luna sobre la Tierra, considerando en este caso la iluminancia, es despreciable respecto de la del Sol. No tiene ningún efecto en la activación de la fotosíntesis y, en consecuencia, en el crecimiento de las plantas.

Una propuesta reducida de la SEA consiste en realizar todas las actividades, pero evitando los cálculos más complejos de las actividades A.6, A.7 y A.8, en las que simplemente se señalará que $\mathrm{g}_{\mathrm{rA}}=\mathrm{g}_{\mathrm{A}}-\mathrm{g}_{\mathrm{L}} \approx 2 \mathrm{GmR} / \mathrm{r}^{3}$ y, sustituyendo sus valores, se encontrará que $\mathrm{g}_{\mathrm{rA}}=2 \mathrm{R} \mathrm{g}_{\mathrm{L}} / \mathrm{r}=\mathrm{g}_{\mathrm{L}} / 30$, lo que nos da una indicación del campo gravitatorio (o aceleración) responsable de las mareas y permite calcular el efecto de las mismas en lagos, plantas, etc.

\section{Conclusiones}

La mayoría de los libros de Física de Bachillerato analizados no tratan las mareas ni tampoco la influencia de la Luna en seres vivos. En cuanto a los manuales universitarios de Física general, salvo los más conceptuales o históricos (Holton y Brush 1976, Carreras et al. 1993, Hewitt 2004), el resto apenas se ocupan de estas cuestiones.

La propuesta didáctica planteada permite, de forma ordenada, realizar los cálculos necesarios que refutan las supuestas influencias de la Luna en aspectos de los seres vivos y de nuestro planeta que no sean las mareas. En efecto, la gravedad de la Luna en la superficie de la Tierra es $3,3 \cdot 10^{-5} \mathrm{~ms}^{-2}$, es decir, 288.000 veces menor que la gravedad terrestre. Esto se traduce en que la gravedad de la Luna sobre cualquier objeto en la superficie de la Tierra es despreciable respecto a la de la propia Tierra. Las mareas tienen lugar debido a la diferencia de gravedad entre la parte del objeto más cercana a la Luna y la más lejana, y su aceleración depende del tamaño del objeto dividido por el cubo de la distancia. Así, la influencia de la Luna en las mareas de los océanos es de $10^{-6} \mathrm{~ms}^{-2}$, pero para un ser vivo de $2 \mathrm{~m}$ de altura, ese valor es de $3 \cdot 10^{-13} \mathrm{~ms}^{-2} \mathrm{y}$, por lo tanto, completamente imperceptible. Lo mismo sucede con la luminosidad de la Luna sobre la Tierra que, como hemos visto, es 128.000 veces menor que el mínimo de la luz solar en un día promedio y, por tanto, no tiene efecto en el crecimiento de las plantas.

Sin embargo, la creencia pseudocientífica de la influencia de la Luna en los seres vivos está muy extendida, no solo entre la población en general sino incluso entre el futuro profesorado de ciencias (Solbes et al. 2018). Como señalan en su metaanálisis Rotton y Kelly (1985), las supuestas relaciones entre las fases de la Luna y el comportamiento pueden atribuirse a 
planteamientos de análisis inapropiados, a no incluir el análisis de otros ciclos (por ejemplo, semanales), y/o a la voluntad de aceptar cualquier desviación del azar como evidencia para un efecto, en este caso, lunar.

Las creencias en el efecto de la Luna, sin aclarar las causas físicas por las que cada influencia debería tener lugar, se producen porque se manipulan los hechos para adaptarlos a las convicciones propias, reforzadas en el grupo, siendo muchas de ellas de carácter popular (tradiciones). Si bien, tal como se ha mostrado al inicio, existen evidencias científicas que desmienten la influencia de la Luna en diferentes fenómenos naturales, hay una serie de sesgos en la ciudadanía que explican la permanencia de ciertas creencias vinculadas a la Luna. En particular, es muy influyente el sesgo retrospectivo, que intenta justificar a posteriori lo que ha ocurrido, y el de confirmación, que busca sólo los datos que confirman nuestras creencias pasando por alto las pruebas que podrían refutarlas (Tokuhama 2011). Este sesgo puede producirse, por ejemplo, si ponemos el foco de atención en los partos que se producen en Luna llena, dejando de lado los producidos en las otras fases.

Por todo ello, el profesorado debe promover el pensamiento crítico como parte esencial de la formación de la ciudadanía. Y para ello es necesario enseñar a detectar falacias de interpretación (respecto a la probabilidad o la causalidad/correlación, etc.), a cuestionar la validez de los argumentos, a rechazar conclusiones no basadas en razones válidas, a detectar tendencias y errores de pensamiento y a evaluar la credibilidad de las fuentes de información. En resumen, a no creer todo lo que se oiga y a pedir pruebas de esos enunciados (Halpern 1998, Solbes 2013, Yager 1993). Por ello es necesario que los libros de Física, y en general los de ciencias, incluyan este tipo de cuestiones.

\section{Agradecimientos}

Este trabajo se ha realizado como parte del proyecto de investigación Propuesta de mejora de la formación del profesorado de ciencias basada en la indagación y modelización en contexto EDU2015-69701-P financiado por el Ministerio de Ciencia e Innovación.

\section{Referencias}

Adushkin V.V., Riabova S.A., Spivak A.A. (2017) Lunar-solar tide effects in the Earth's crust and atmosphere. Izvestia Physics Solid Earth, 53, 565-580.

Andrés D.C., Antón J.L., Barrio J. (2009) Física $2^{\circ}$ Bachillerato. Madrid: Editext

Alonso M., Finn E.J. (1995) Física. Wilmington: Addison Wesley.

Anglés J.M. (1984) Influencia de la Luna en la agricultura y otros temas de especial interés para el campesino y gentes de la ciudad. Lleida: Dilagro-Ediciones.

Arliss J.M., Kaplan E.N., Galvin S.L. (2005) The effect of the lunar cycle on frequency of births and birth complications. American Journal Obstetric Gynecology, 192, 1462-1464.

Armario M., Oliva J.M., Jiménez-Tenorio N. (2019) El fenómeno de las mareas y su explicación. Alambique. Didáctica de las Ciencias Experimentales 95, 31-38.

Armario M., Jiménez-Tenorio N., Oliva J.M. (2021) Tratamiento del fenómeno de las mareas en libros de texto de Secundaria. 29 Encuentros de Didáctica de las Ciencias Experimentales, 1350-1357.

Bakulin P.I., Kononovich E.V., Moroz V.I. (1987) Curso de Astronomía General. Moscú: Mir.

Carreras C., Fernández-Rañada A., García J.J., Yuste M. (1993) Física básica. Madrid: Alianza. 
Casas J. (1985) Óptica. Universidad de Zaragoza.

Corrochano D., Gómez-Gonçalves A., Sevilla J., Pampín-García S. (2017) Ideas de estudiantes de instituto y de universidad acerca del significado y el origen de las mareas. Revista Eureka sobre Enseñanza y Divulgación de las Ciencias 14, 353-366.

Dalmau J.F., Pérez M., Satoca J., Tejerina F. (2007) Física $2^{\circ}$ Bacbillerato. Madrid: Anaya.

Feynman R.P., Leighton R.B., Sands M. (1971) The Feynman Lectures on Physics Volumen 1. Bogotá: Fondo Educativo Interamericano.

Gerthsen C., Kneser H.O., Vogel H. (1979) Física. Madrid: Dossat y Springer Verlag.

Gettys W.E., Keller F.J., Skove M.J. (1991) Física clásica y moderna. Aravaca: McGraw-Hill.

Giancoli D.C. (1985) Física. Principios y aplicaciones. Barcelona: Reverté

Gupta R., Nolan D.R., Bux D.A., Schneeberger A.R. (2019) Is it the moon? Effects of the lunar cycle on psychiatric admissions, discharges and length of stay. Swiss medical weekly 149(1718).

Halliday D., Resnick R., Krane K.S. (2007) Física. México: Patria.

Halpern D. (1998) Teaching critical thinking for transfer across domains. American Psychologist 53(4), 449-455.

Hewitt P.G. (2004) Física Conceptual. México: Pearson Education.

Holton G., Brush S. (1976) Introducción a los Conceptos y Teorías de las ciencias físicas. Barcelona: Reverté.

Lipner S.R., Scher R.K. (2017) Nail growth evaluation and factors affecting nail growth. In: Humbert P, Fanian F, Maibach H, et al, eds. Agache's Measuring the Skin. Cham, Switzerland: Springer, 1-15.

Lorente S., Sendra F., Enciso E., Quílez J., Romero J. (2009) Éter. Física. Valencia: ECIR.

Marco-Gracia F.J. (2019) The influence of the lunar cycle on spontaneous deliveries in historical rural environments. European Journal Obstetric Gynecology Reproduction Biology 236, 22-25.

Martínez V.J., Miralles J.A., Marco E., Galadí-Enríquez D. (2005) Astronomía fundamental, Universitat de Valencia

Mayoral O., Solbes J., Cantó J., Pina T. (2020) What Has Been Thought and Taught on the lunar Influence on Plants in Agriculture? Perspective from Physics and Biology. Agronomy 10, 955.

Messenger A. G. (1993) The control of hair growth: an overview. Journal of Investigative Dermatology 101(1), S4-S9.

Montesinos J., Cortes J., Arnau A., Sanchez J. A., Elmore M., Macia, N., Bosch J. (2013) Barcelona baby boom: does sporting success affect birth rate? Bmj 347.

Morgan E. (2001) The moon and life on earth. Earth Moon Planets 85-86, 279-290.

Ortuño M. (1996) Física para biología, medicina, veterinaria y farmacia. Barcelona: Crítica.

Rackham T. (1968) Moon in Focus. Oxford: Pergamon.

Raven, J.A., Cockell, C.S. (2006). Influence on photosynthesis of starlight, moonlight, planetlight, and light. Astrobiology 6, 668-675. 
Restrepo J. (2004) La Luna: el sol nocturno en los trópicos y su influencia en la agricultura. Managua: Servicio de Información Mesoamericano sobre Agricultura Sostenible.

Rotton J., Kelly I.W. (1985) Much Ado About the Full Moon: A Meta-Analysis of LunarLunacy Research. Psychological Bulletin 97, 286-306

Sears F. W., Zemansky M.,Young H.D., Freedman, R.A. (2009) Física Universitaria. Mexico: Pearson Education.

Smith F.G., Thomson J.H. (1989) Optics. Hoboken, NJ: Wiley.

Solbes, J. (2013) Contribución de las cuestiones sociocientíficas al desarrollo del pensamiento crítico (I): Introducción. Revista Eureka sobre Enseñanza y Divulgación de las Ciencias 10, 1 10.

Solbes J., Palomar R., Domínguez-Sales M. C. (2018) To what extent do pseudosciences affect teachers? A look at the mindset of science teachers in training. Mètode Science Studies Journal, 8, 188-195.

Tipler P.A. (1992) Física. Barcelona: Reverté.

Torres, N.Y., Solbes, J. (2016). Contribuciones de una intervención didáctica usando cuestiones sociocientíficas para desarrollar el pensamiento crítico. Enseñanza de las ciencias. 34 (2), 43-65.

Tokuhama T. (2011) Mind, Brain and Education Science. A comprehensive guide to the new brain-based teaching. New York: Norton \& Company.

Vidal M.C., Sanchez D. (2016) Física $2^{\circ}$ Bachillerato. Madrid: Santillana.

Viiri, J. (2000) Students' understanding of tides. Physics Education 35, 105-105.

Yager R.E. (1993) Science and critical thinking. In Clarke, J.H., Biddle, A.W., Eds. Teaching Critical Thinking: Reports from Across the Curriculum. Englewood Clis, NJ: Prentice Hall.

Yavorski B.M., Detlaf A.A. (1977) Manual de física, Moscú: Mir. 